\title{
Diagnostic robust generalised potentials based on GM6 to identify high leverage points in simultaneous regression model
}

\begin{abstract}

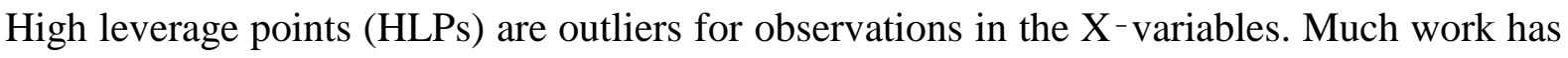
been focused on the detection of high leverage points (HLPs) in multiple linear regression but no research has been done on the detection of HLPs in simultaneous equation models (SEM). Like multiple linear regression, the detection of HLPs in SEM is also important because of their responsibility for producing inefficient estimates. Since no such research has been done, we make the first attempt to propose a diagnostic method by adapting the diagnostic robust generalised potential (DRGP) to identify HLPs in SEM. Numerical examples and simulation results indicate that our proposed method is very successful in identifying HLPs in SEM.
\end{abstract}

Keyword: Generalised potential; High leverage points; Simultaneous equation models; 2SLS 